\title{
Molecular insights on potato yellow vein crinivirus infections in the highlands of Colombia
}

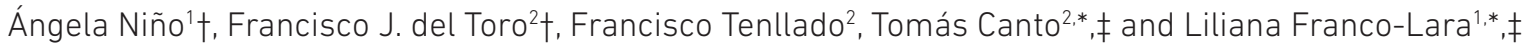

\begin{abstract}
Potato yellow vein virus (PYVV) was detected in potatoes grown in the Central highlands, north of Bogotá ( $3000 \mathrm{~m}$ altitude), Colombia. At this altitude viral whitefly vectors are largely absent, but infection persists because of the use of uncertified tubers. Plants with typical PYVV-induced yellowing symptoms, as well as with atypical yellowing or non-symptomatic symptoms were sampled at three separate geographical locations. PYVV presence was assessed by RT-PCR, and several plants were subjected to high-throughput sequencing (HTS) of their small RNA (sRNA) populations. Complete or almost complete sequences of four PYVV isolates were thus reconstructed, all from symptomatic plants. Three viral isolates infected plants singly, while the fourth co-infected the plant together with a potyvirus. Relative proportions of sRNAs to each of the three crinivirus genomic RNAs were found to remain comparable among the four infections. Genomic regions were identified as hotspots of sRNA formation, or as regions that poorly induced sRNAs. Furthermore, PYVV titres in the mixed versus single infections remained comparable, indicating an absence of synergistic/antagonistic effects of the potyvirus on the accumulation of PYVV. Daughter plants raised in the greenhouse from tubers of the infected, field-sampled plants displayed mild PYVV infection symptoms that disappeared with time, demonstrating the occurrence of recovery and asymptomatic infection phenotypes in this pathosystem.
\end{abstract}

\section{INTRODUCTION}

Potato yellow vein virus (PYVV) causes a disease known as potato yellow vein disease (PYVD) in potato crops in Colombia, Ecuador, Perú and Venezuela [1], with crop losses estimated at $30-50 \%$, because of decreases in the size and number of tubers produced [2-4]. In Colombia, two species of potato are cultivated commercially, Solanum phureja and S. tuberosum, the first a diploid species and second tetraploid [5]. Despite this and other morphological and physiological differences, these two species are genetically close, such that some authors consider them as part of the same species [6]. In infected plants, the yield reductions are associated with the presence of symptoms, such as initial yellowing of the secondary and tertiary leaf-veins that later spreads throughout and around the foliar veins, covering the lamina. These bright yellow leaf symptoms cause a reduction in the photosynthetic capacity and vigour of the plants [3]. The only known vector of PYVV is the greenhouse whitefly, Trialeurodes vaporariorum Westwood (Hemiptera: Aleyrodidae) $[1,4]$, but it can also be transmitted through infected seed-tubers $[1,5]$. It has also been reported that in the field, a proportion of the infected plants may not display symptoms $[1,7,8]$ and that yields of such plants are not reduced [3].

PYVV is a member of the genus Crinivirus, family Closteroviridae $[1,9]$. Criniviruses are one of the three genera described for Closteroviridae, and include phloem-limited viruses of positive-sense, bipartite and, in the case of PYVV, tripartite RNA genomes, that are transmitted semi-persistently by hemipteran whiteflies of the genera Trialeurodes or Bemisia [10, 11]. All Crinivirus share two characteristic genomic features: first, they have a replication module that contains domains for a papain-like leader proteinase (L-Pro), a

Received 15 September 2020; Accepted 30 April 2021; Published 07 June 2021

Author affiliations: 'Faculty of Basic and Applied Sciences, Universidad Militar Nueva Granada, Km 2 via Cajicá-Zipaquirá, Cajicá, Cundinamarca, Colombia; ${ }^{2}$ Department of Microbial and Plant Biotechnology, Center for Biological Research, CIB-CSIC, Ramiro de Maeztu 9, Madrid 28040, Spain.

*Correspondence: Tomás Canto, tomas.canto@cib.csic.es; Liliana Franco-Lara, liliana.franco@unimilitar.edu.co

Keywords: PYVV infections in potato; crinivirus genome organization; crinivirus and potyvirus mixed infections; vertical transmission of criniviruses in potato.

Abbreviations: HTS, high-throughput sequencing; PYV, potato virus Y; PYVD, Potato yellow vein disease; PYVV, potato yellow vein virus; RPKM, readsper-million-per-kilobase-of-template; RPM, reads-per-million; sRNA, small RNA.

MT319730, MT334622, MT334624, MT522445.

†These authors contributed equally to this work

$\ddagger$ These authors also contributed equally to this work

One supplementary table and two supplementary figures are available with the online version of this article.

$001604 @ 2021$ The Authors 
(a)
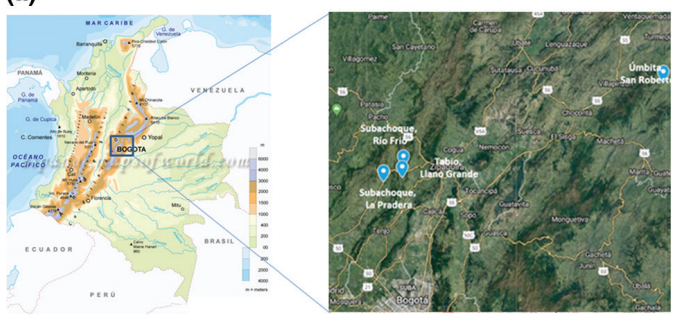

(b)

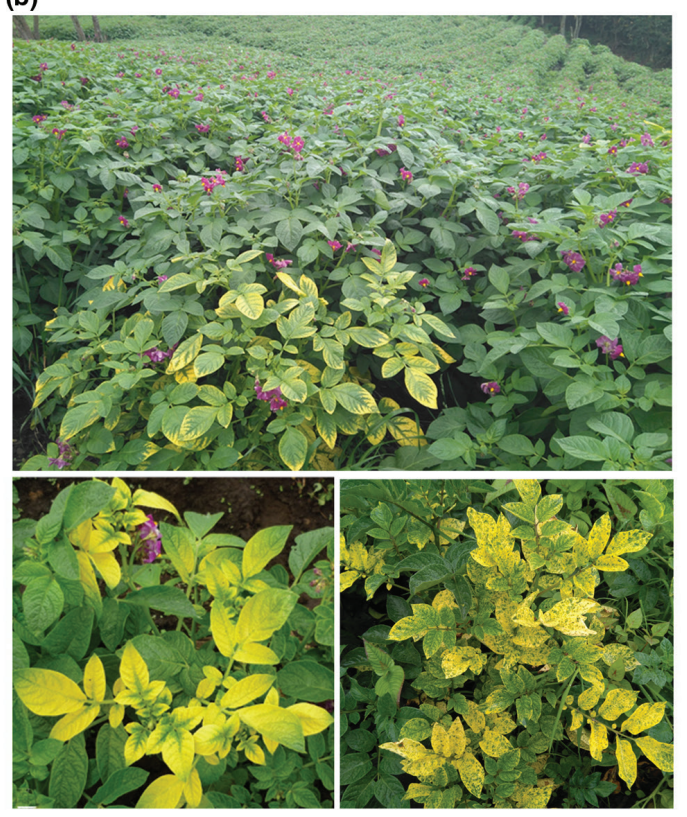

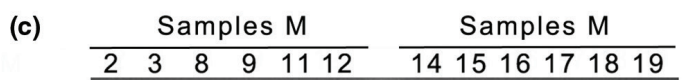

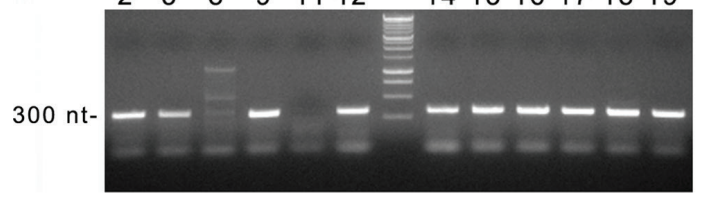

Fig. 1. Sampling at three different sites in the Central Colombian highlands of potato fields that contained plants with PYVD-like symptoms, description of symptoms and detection of PYVV presence by RT-PCR. (a) The geographical highland sites where samples were collected: Subachoque-Rio Frío (5.018750, -74.093000), Tabio-Llano Grande (4.9936667, -74.0961389) and Úmbita-San Roberto (5.211971. -73.482558). (b) Upper panel, characteristic PYVD symptoms caused by PYVV in a Solanum phureja plant at the forefront of the image, consisting of mild yellowing of the leaf blade but confined around foliar veins; lower panel to the left, stronger PYVD symptoms characterized by extended and intense yellowing in sink areas of leaves in a S. phureja plant; lower panel to the right, atypical strong symptoms characterized by an extended mosaic of green and intense areas of yellow in Solanum tuberosum. (c) Assessment of the presence of PYVV in 12 samples by the amplification of a $300 \mathrm{nt}$ fragment from genomic RNA 1 by RT-PCR.

methyltransferase (MTR), a helicase (HEL) [open reading frame 1a (ORF1a)] and an RNA-dependent RNA polymerase [RdRp (ORF1b)] [10]. Second, downstream of this module, there is a hallmark gene array encoding a putative small hydrophobic protein, a heat-shock protein homologue (Hsp70h), a protein of between 50 and $60 \mathrm{kDa}$ depending on the virus, the viral coat protein $(\mathrm{CP})$ and a minor $\mathrm{CP}(\mathrm{CPm})$ respectively, an arrangement unique among members of the genus Crinivirus [10, 12].

The first genome of PYVV was fully sequenced [9] and was found to comprise three separate RNAs: RNA 1, RNA 2 and RNA 3, of $\sim 8,5.3$, and $3.8 \mathrm{~kb}$, respectively. RNA 1 contains the replication module and codes for three ORFs: ORF 1a and $b$, and the conserved L-Pro, MTR, HEL and RdRp domains; and p7, a small hydrophobic protein. Additionally, it codes for a small putative protein of $6.9 \mathrm{kDa}$. In RNA 2, five ORFs were predicted: Hsp70h, p7, p60, p10 and CP (Fig. 1a), which correspond to the conserved gene array of closteroviruses. RNA 3 encodes three potential ORFs: p4, CPm and p26, also consistent with the closterovirus gene array [9]. In addition to the initial Peruvian isolate sequenced [9], full PYVV sequences were later obtained in Colombia from infected potatoes (Solanum phureja) [13], tomatoes (Solanum lycopersicum) [14] and lulo plants (Solanum quitoense) [15].

PYVV is also known to infect weed species of the genus Polygonum, in asymptomatic infections [1]. PYVV has been reported to occur in the field also in mixed infections with potyviruses in potato [16].

In this work we performed high throughput sequencing (HTS) of the small RNA (sRNA) populations present in potato plants sampled at three different geographical locations in the Colombian highlands. Single PYVV infections as well as a mixed infection with the potyvirus potato virus Y (PVY) were found. We reconstructed the genomes of the infecting viruses and determined the contribution to sRNA induction of each of the three PYVV genomic RNAs, and identified genomic regions that were hotspots to sRNA formation and others that induced them poorly. We determined that in this pathosystem co-infection with PVY had no synergic effect on the accumulation of PYVV. In progenies raised from tubers from PYVV-infected plants under controlled glasshouse conditions, we also observed the occurrence of recovery and of asymptomatic infection phenotypes.

\section{METHODS}

\section{Sampling of plant material from potato fields}

Leaves $(\sim 5 \mathrm{~g})$ from potato plants from highland crops were collected in April 2018 at three locations of the Colombian departments of Cundinamarca and Boyacá, in Central Colombia: Subachoque-Rio Frío (5.018750, -74.093000), Tabio-Llano Grande (4.9936667, -74.0961389) and ÚmbitaSan Roberto (5.211971, -73.482558). Leaves and tubers from 20 plants showing either typical PYVD yellowing symptoms, atypical yellowing symptoms or no symptoms at all were collected (Table 1, Fig. 1).

One to four tubers were also collected from each of the 12 sampled plants, including plants with PYVD symptoms, atypical yellowing or without symptoms, for the analysis of progenies raised from them. Tubers were initially stored 
Table 1. Geographical origin, symptoms and analysis for PYVV presence in potato leaves from the species Solanum phureja and S. tuberosum subsp. andigena collected in field crops in the Central highlands of Colombia

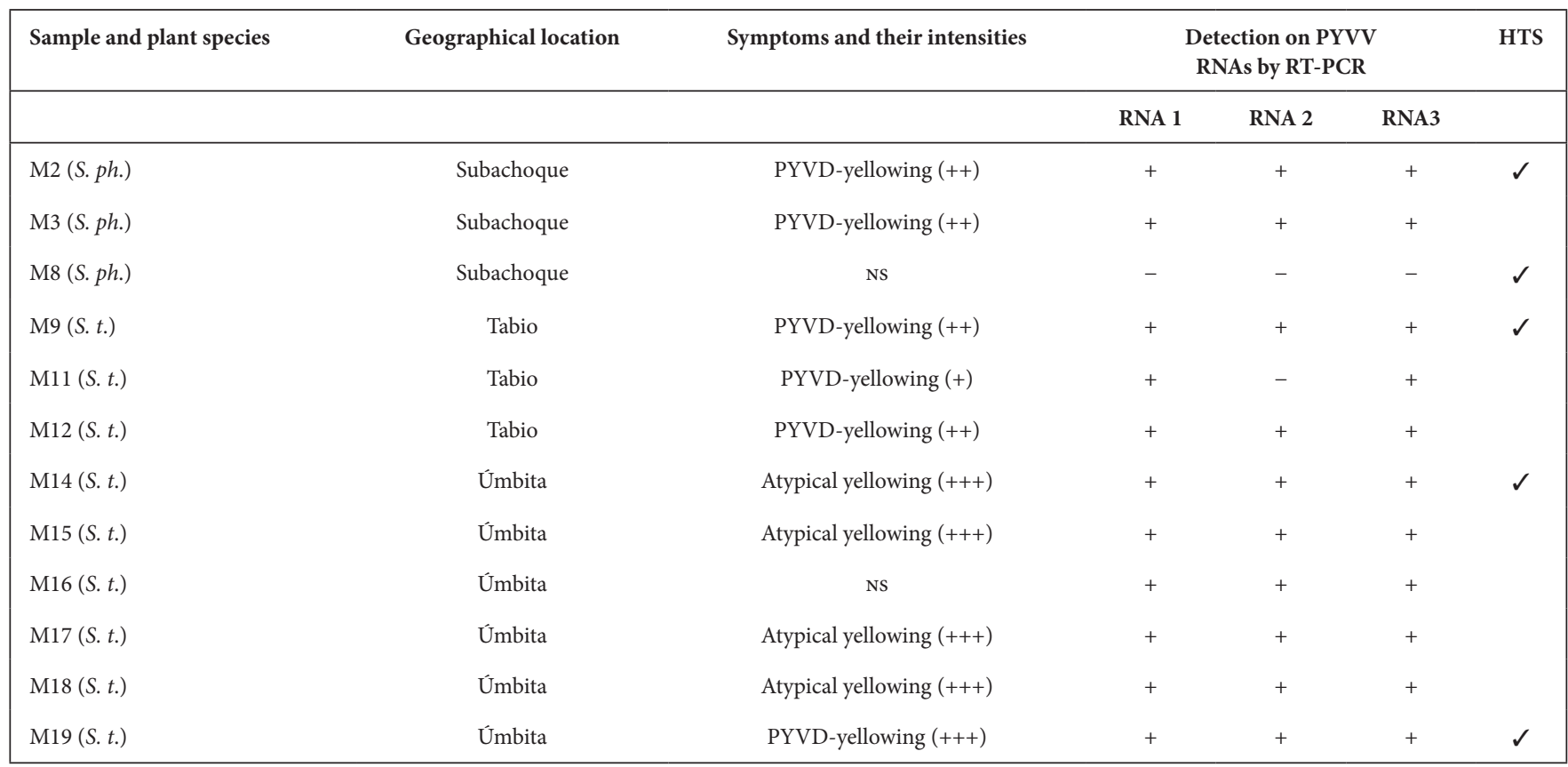

S. t., Solanum tuberosum subsp. andigena. S. ph., Solanum phureja. PYVD-yellowing, typical PYVV symptoms, with yellowing of the leaf lamina, with green midribs and veins. Atypical yellowing, bright yellow/green mosaic leaf symptoms. Symptom intensities: NS, no symptoms; +, very mild symptoms; ++, mild symptoms; +++, strong symptoms. $\checkmark$ Samples that were subjected to sequencing of their sRNA populations by HTS.

in paper bags in the darkness, at room temperature, for 2 months. When tubers began to shoot, they were planted in black plastic bags containing $10 \mathrm{~kg}$ of sterile soil fertilized once with a mineral fertilizer $\left(\mathrm{N}: 15 \%, \mathrm{w} / \mathrm{w} ; \mathrm{P}_{2} \mathrm{O}_{5}\right.$ : $15 \%, \mathrm{w} / \mathrm{w}$; and $\mathrm{K}_{2} \mathrm{O}: 15 \%$, w/w). The plastic bags containing the progeny plants were kept in insect-free mesh cages, inside a plastic greenhouse at the Campus of Universidad Militar Nueva Granada in Cajicá, Cundinamarca, until senescence (around 16 weeks post-emergence for $S$. phureja and 20 weeks post-emergency for $S$. tuberosum). Plants were watered three times a week. Temperatures were semicontrolled by opening and closing the greenhouse windows, and during the lifespan of these plants it ranged from $10^{\circ} \mathrm{C}$ at night to $30^{\circ} \mathrm{C}$ during the day. For all progeny plants, symptoms were visually assessed weekly, and the presence of the virus was tested by RT-PCR.

\section{Detection of PYVV in leaf samples by RT-PCR}

Total RNA was extracted from the leaves sampled in the field using Invitrogen Trizol Reagent (Invitrogen) following the instructions from the manufacturer. Briefly, for each extraction $1 \mathrm{~g}$ of leaf lamina including secondary and tertiary veins was ground to powder in liquid nitrogen, and $5 \mathrm{ml}$ of TRIzol Reagent (Invitrogen) was then added. Extracts were incubated under strong shaking at room temperature for $10 \mathrm{~min}$ and then centrifuged at $10000 \mathrm{~g}$ for $10 \mathrm{~min}$. Aqueous and organic phases were resolved by the addition of $2 \mathrm{ml}$ of chloroform and by centrifugation at $10000 \mathrm{~g}$ for $10 \mathrm{~min}$. The aqueous phase was then collected and precipitated with
1 volume of 2-propanol overnight at $4{ }^{\circ} \mathrm{C}$. Total RNAs were pelleted by centrifugation at $10000 \mathrm{~g}$ for $15 \mathrm{~min}$ at $4{ }^{\circ} \mathrm{C}$, and the pellets were washed with $1 \mathrm{ml}$ of $100 \%$ ethanol followed by a second wash with $1 \mathrm{ml}$ of $75 \%$ ethanol. RNA pellets were dissolved in sterile water, and their concentrations and qualities were analysed with Nanodrop (Thermofisher Scientific) and Experion (Bio-Rad) instruments following the manufacturers' instructions.

For the reverse transcription from total RNA samples of segments of the PYVV viral RNAs, a mixture of three reverse primers (at 2 pmol each, Table S1, available in the online version of this article) was used, using Superscript III Reverse Transcriptase, following the manufacturer's instructions (Invitrogen). Amplification by PCR of the thus amplified cDNA fragments from each of the three genomic viral RNAs was then performed with the appropriate oligos (Table S1).

\section{HTS of SRNA populations in the sampled plants}

To analyse the sRNA populations present in the sampled leaves, separate deep sequencing events were performed, one per individual plant. Total RNAs were extracted from $\sim 1 \mathrm{~g}$ of leaf tissue with TRIzol reagent following the instructions of the manufacturer. Fifty micrograms of total RNAs was then used for sequencing of their sRNA populations (reads of 18-50 nt in length). RNA sequencing was performed by Fasteris SA: briefly, the sRNA fractions were resolved from the total RNA samples using TruSeq 


\section{sRNA proportions in samples:}
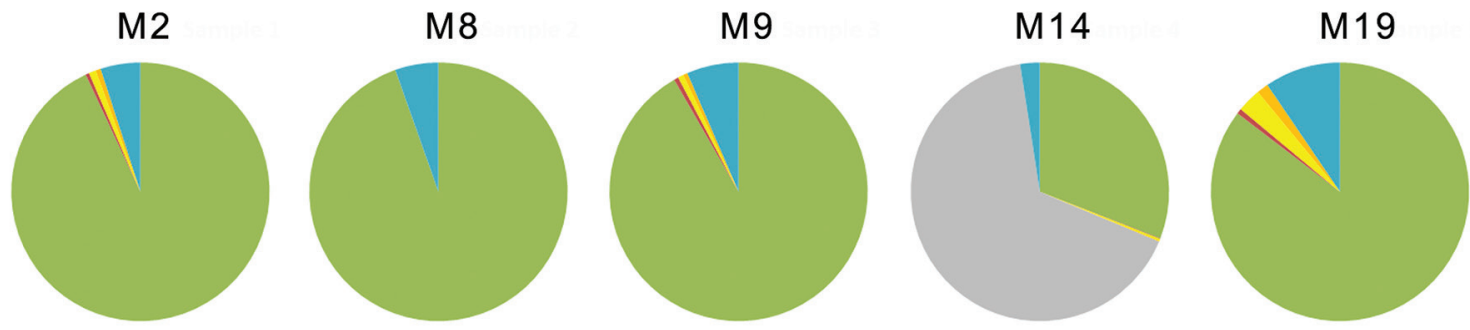

Potato sequences

-PYVV RNA 1

PYVV RNA 2

-PYVV RNA 3

『VY

Potato 2 nd alignment (1 mismatch)

Fig. 2. Pie charts of the relative proportions of SRNA reads found in each of the five leaf samples analysed by next generation sequencing. Reads (reads-per-million; RPM) are classified as being of either plant (green and blue colours) or non-plant origin (in this case all of them of viral origin: brown, orange, yellow or grey colours). Samples M2 (Solanum phureja), M9 (Solanum tuberosum), M14 (S. tuberosum) and M19 (S. tuberosum) (symptomatic) contained sRNAs to PYVV. Sample M14 contained in addition sRNAs to PVY. Sample M8 (S. phureja) (asymptomatic) had only sRNAs of plant sequence, and was therefore uninfected.

Small RNA kits (Illumina), and subjected afterwards to $5^{\prime}$ cap removal, followed by single-stranded ligation of $3^{\prime}$ and $5^{\prime}$ indexed adapters. Reverse transcription and PCR amplification were then performed to generate the cDNA libraries that were multiplexed and sequenced in one lane of $50 \mathrm{bp}$ single-end runs in a NextSeq 100M 1×50 Illumina sequencer.

\section{Analysis of the sRNA reads generated by HTS}

After adapter removal, sequence reads from the generated RNA libraries of 18-50 nt in length were computationally processed using our own Perl scripts. Reads were then mapped (full identity) using Bowtie [17] against the potato (S. phureja) genome and transcriptome (potato_dm_v404_ all_pm_un.fasta, and PGSC_DM_v3.4_transcript.fasta files respectively; http://solanaceae.plantbiology.msu.edu/pgsc_ download.shtml) [18]. Given the genetic proximity between $S$. phureja and S. tuberosumm $[5,6]$ the same reference sequence derived from $S$. phureja was used. Reads that did not match potato sequences were re-mapped against the potato genome, allowing for one mismatch and most of them were identified as being of plant origin using these methods (shown in blue colour in the pie charts of Fig. 2).

In order to obtain the genomic sequences of the viruses infecting those samples, the remaining non-potato matching sequences were mapped against known plant virus sequences using the free software package VirusDetect [19]. This program generates contigs of viral sequences that match viral sequences in gene banks: in our case of PYVV in four of our potato samples, and of an additional potato virus Y (PVY; genus Potyvirus) potyvirus in one of the four samples. Some of those contigs covered large or complete viral genomic RNAs, while others were much shorter, incomplete genomic sequences. The incomplete contigs were further assembled based by comparison among the contigs themselves and with published viral accessions (KR998193 for PYVV RNA1; AJ557129 for PYVV RNA 2; AJ508757 for PYVV RNA 3), with the assembly tool of the Program Vector NTI Advance 11.0 (Invitrogen). Nine genomic PYVV viral RNAs were partially reconstructed in this way (data not shown). When reconstructed PYVV genome sequences were incomplete (Fig. 3), the small percentages of missing nucleotides were replaced with the corresponding nucleotide sequences from those accessions, to allow for further analysis.

The HTS raw and processed data, the latter consisting in the 13 reconstructed viral genomic RNAs detected and the distribution through their genomes of sRNA reads, have been uploaded into the Gene Expression Omnibus (series accession number GSE157752; GEO, http://www.ncbi.nlm. nih.gov/geo). Three sequences of PYVV genomic RNAs that were complete, as well as the complete sequence of the PVY isolate, were uploaded into GenBank, with accession numbers MT334622 (Sample M2, PYVV RNA1), MT334624 (Sample M9, PYVV RNA2), MT319730 (Sample M19, PYVV RNA 1) and MT522445 (Sample M19, PVY).

Sorting of sRNA reads by length, counting of total and of unique sequences, and mapping of reads over the reconstructed viral genomes were all made with different Perl scripts developed by our laboratory.

\section{Relative quantification of viral RNAs by semi- quantitative RT-PCR}

Semi-quantitative RT-PCR amplifications were performed to measure relative viral titres in different plants. Total RNAs from each plant were first treated with TURBO DNase following the manufacturer's instructions 

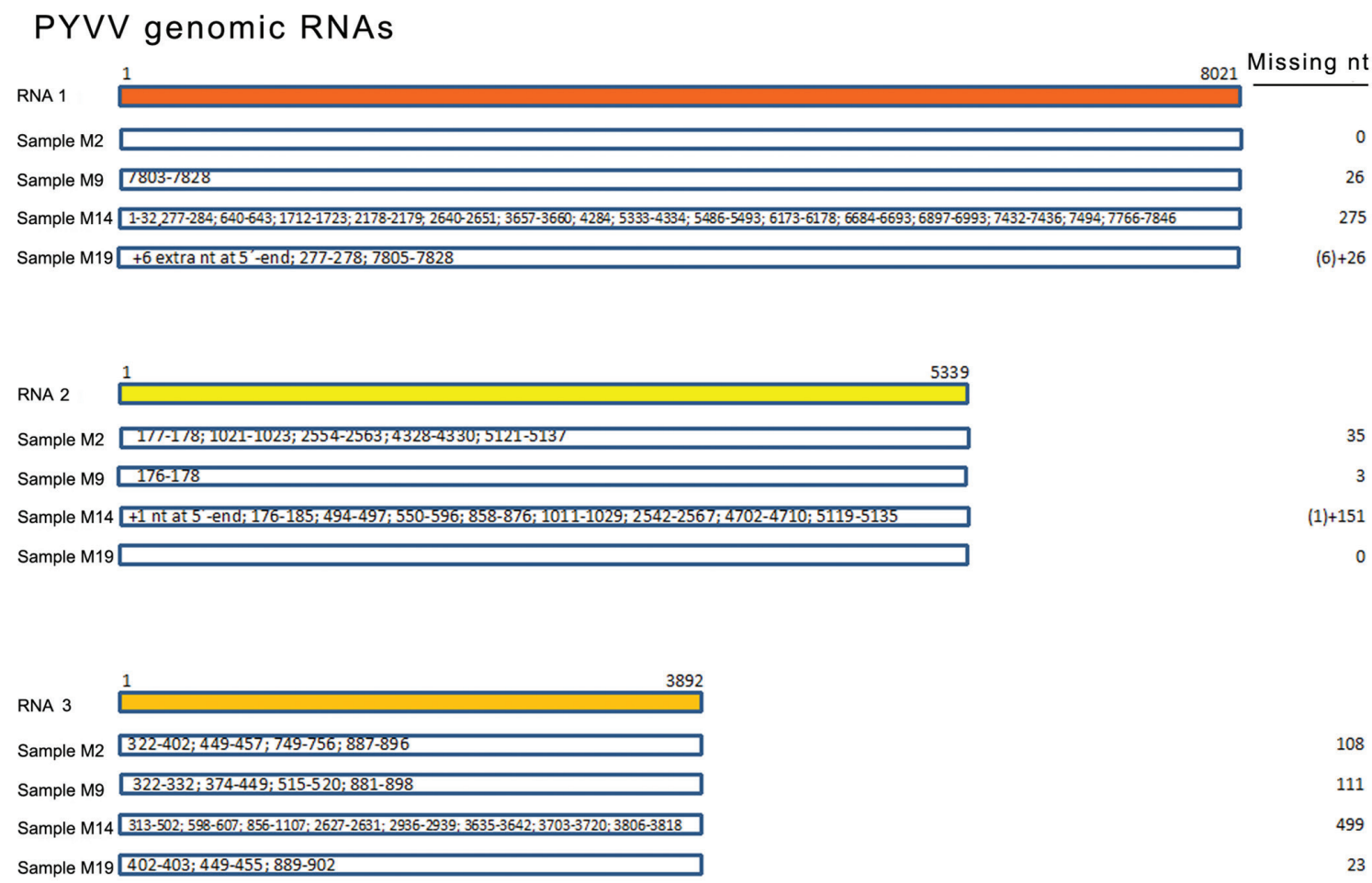

108

111

499

0 6

275

$+26$ (1)

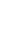
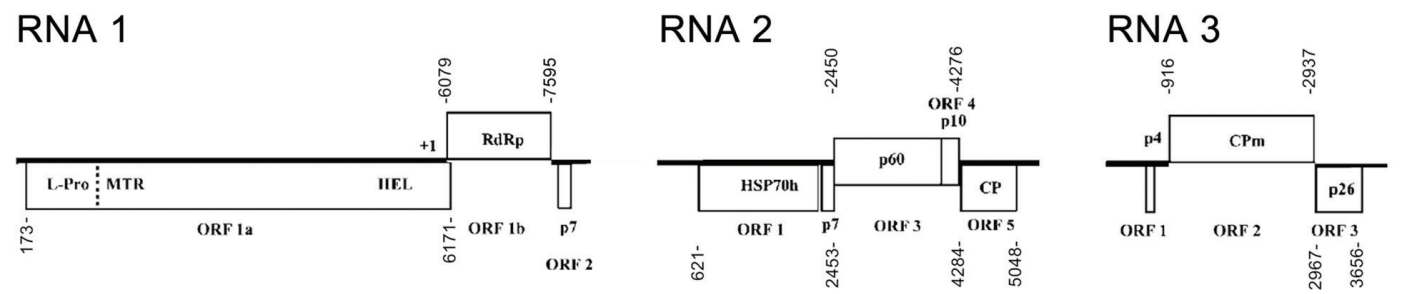

Fig. 3. Reconstruction of the genomic RNAs of PYVV infecting field potato plants, using HTS of sRNA populations. Two complete genomic sequences were assembled from contigs of the sRNA reads [PYVV RNA 1 in Sample M2 (Solanum phureja) and PYVV RNA 2 in Sample M19 (Solanum tuberosum)]. The other genomic sequences were incomplete, and the absent nucleotide segments are indicated individually. In the case of single PYVV infections [Samples M2, M9 (S. tuberosum), and M19] the average number of missing nucleotides was low ( 0.3\% of the total for RNA 1, $0.6 \%$ for RNA 2 and $2.8 \%$ for RNA 3). In the case of the mixed infection with PVY in sample M14 (S. tuberosum), those percentages were larger because of the reduced number of reads to PYVV sRNAs obtained (3.4\% for RNA 1, 2.8\% for RNA 2 and $12.8 \%$ for RNA 3). Note that the unidentifed nucleotide segments in different samples often map to the same regions of the viral RNA, indicating that the absence of continua of overlapping sRNA reads in those segments needed for contig assembly is conserved through the different infections. The reconstructed genomic PVY sequence was, by contrast, complete (not shown).

(Thermofisher Scientific). Reverse transcriptions into cDNAs of PYVV fragments from each of the three viral genomic RNAs and from a ribosomal 18S RNA housekeeping fragment were performed using a mixture (2 pM each) of the four appropriate reverse oligos (Table S1). The resulting cDNAs were then amplified in triplicate tubes using Phusion polymerase (Finnzymes) with the following cycling programme: $98^{\circ} \mathrm{C}$ for $1 \mathrm{~min}$, for one cycle; $98^{\circ} \mathrm{C}$, $10 \mathrm{~s} / 54^{\circ} \mathrm{C}, 10 \mathrm{~s} / 72^{\circ} \mathrm{C}, 10 \mathrm{~s}$, for 40 cycles, with one of the triplicate PCR tubes being retrieved sequentially at cycles 25, 32 and 40.

\section{RESULTS}

\section{Symptom characterization and detection of PYVV infection}

Leaves and tubers from 19 plants of S. phureja or S. tuberosum sp. andigena were collected in 2018 from symptomatic field potato crops at three different sites in the Central Colombian highlands (Fig. 1a). Some of the sampled plants showed characteristic PYVD symptoms in leaves to different degrees of intensity, as had been described before $[1,5]$, whereas others had atypical yellowing symptoms described as unevenly 


\section{sRNA proportions in samples:}
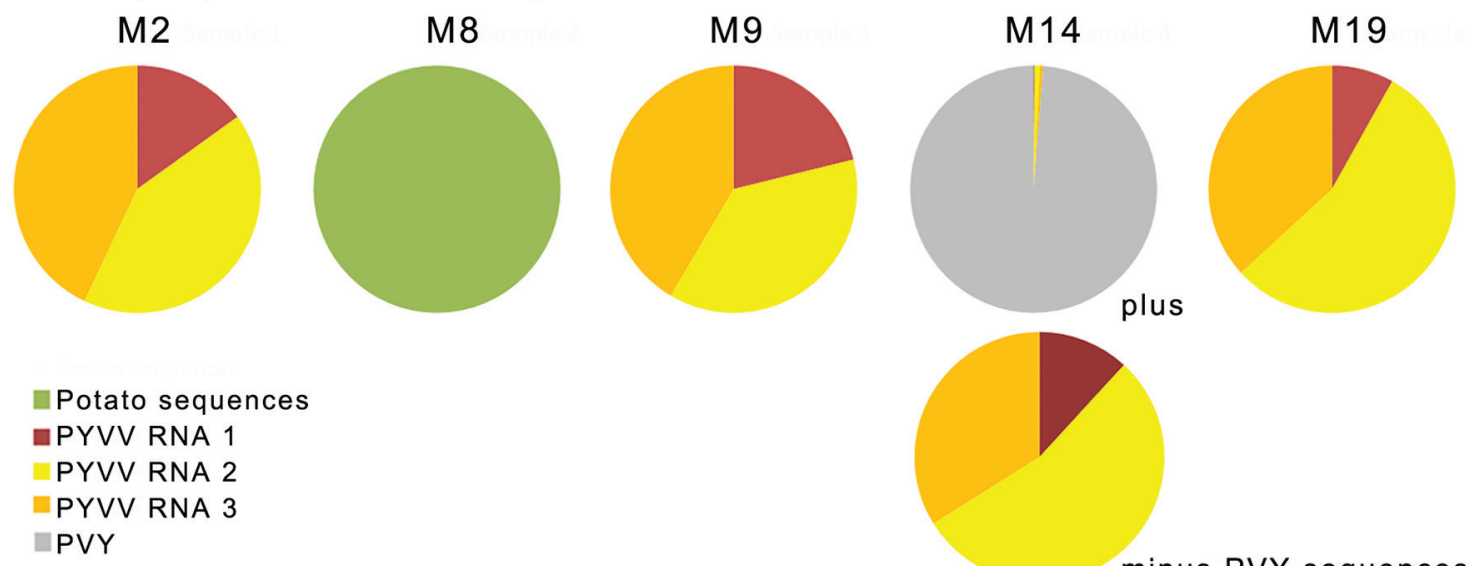

Fig. 4. Pie charts showing the relative proportions of sRNA reads found in each of the five samples analysed by next generation sequencing, and relative to the lengths of the sequences from which they originated. Reads (RPKM) are classified as being of either plant (green colour) or non-plant origin (in this case almost all of them being of viral origin: brown, orange and yellow colours for those with PYVV genomic RNAs 1, 2 and 3 sequence, respectively, or grey for those with PVY genomic RNA sequence). Relative proportions among the reads to each of the genomic RNAs of the phloem-confined PYVV remained fairly stable in the four samples that were infected with the virus: M2 (Solanum phureja), M9 (Solanum tuberosum), M14 (S. tuberosum) and M19 (S. tuberosum), even in the case of sample M14, where it co-infected the plant with the potyvirus PVY, a systemic virus not confined to the vasculature.

distributed green patches on yellow leaf laminas (example shown in Fig. 1b, upper panel and lower right panel). Finally, some of the sampled plants were asymptomatic (not shown).

Of the sampled plants, 12 that were representative of the different symptoms observed (or of their absence in the vicinity of symptomatic plants) were tested for PYVV infection by RT-PCR (Table 1) with primers for the three viral genomic RNAs (Table S1). PYVV presence was confirmed in all the plants with yellowing symptoms (Table 1).

\section{Characterization of SRNA populations}

To gain further insight into PYVV infections in this pathosystem, we sequenced by HTS the sRNA populations present in samples M2, M9 and M19 that showed PYVD symptoms, another sample M14 that showed atypical yellowing symptoms, and in asymptomatic sample M8 (Table 1). Between 25 and 36 million total sRNA reads (18-50 nt) were obtained for each of the five samples, in all cases the most abundant being those 21,22 and $24 \mathrm{nt}$ in length (data not shown). Reads were classified as either of plant sequence (Fig. 2, green and blue sections of pie charts representing the proportion of sRNA reads in reads-per-million, RPM; the blue section originating from a second alignment round that allowed up to one mismatch between the sRNA read and genomic sequence) or of non-plant sequence. Of the reads of non-plant sequence, a few matched other organisms, such as Fungi or Diptera but their numbers were residual and may have originated from spores or insect eggs present on the surfaces of the leaves (data not shown). The vast majority of non-plant reads matched viral sequences (Fig. 2, remaining colours in corresponding pie charts). One of the five samples analysed was uninfected (asymptomatic sample M8), four of the samples contained only the three PYVV genomic RNAs (M2, M9, M14 and M19), while sample M14 contained reads to the three PYVV genomic RNAs and also a large proportion of reads to the potyvirus PVY (Fig. 2). In the case of the latter mixed infection, the number of total reads to each of the genomic RNAs of PYVV was around an order of magnitude lower than those generated to the PYVV genomic RNAs in the three single infections (Fig. 2, and data not shown).

When the proportions of plant and non-plant sRNA reads were compared to the length of the sequences that triggered them, viral template sequences were shown to be highly immunogenic (Fig. 4, represented as pie charts of the proportion of sRNA reads in reads-per-million-per-kilobase-of-template, RPKM), indicating that they triggered synthesis by the plant of relatively large amounts of sRNAs. Interestingly, the relative proportions of reads to each of the genomic viral RNAs of PYVV remained broadly similar among the four samples analysed, even when in the mixed infection with PVY (Fig. 4), indicating that those relative proportions were a stable trait of infection by PYVV in this pathosystem. Reads to PYVV RNA 1 were the less frequent, being between a third and a half of those to either RNAs 2 or 3 , even though genomic RNA 1 has the largest size of the three (Fig. 4, brown vs. yellow and orange areas in the pie charts).

\section{Reconstruction of the genomic sequences of the viruses present in the sampled plants, and some inferred molecular properties}

Assembly of the contigs of sRNA reads of viral sequence generated led to the complete or almost complete reconstruction of 


\section{Distribution of sRNA reads on the PYVV genome}
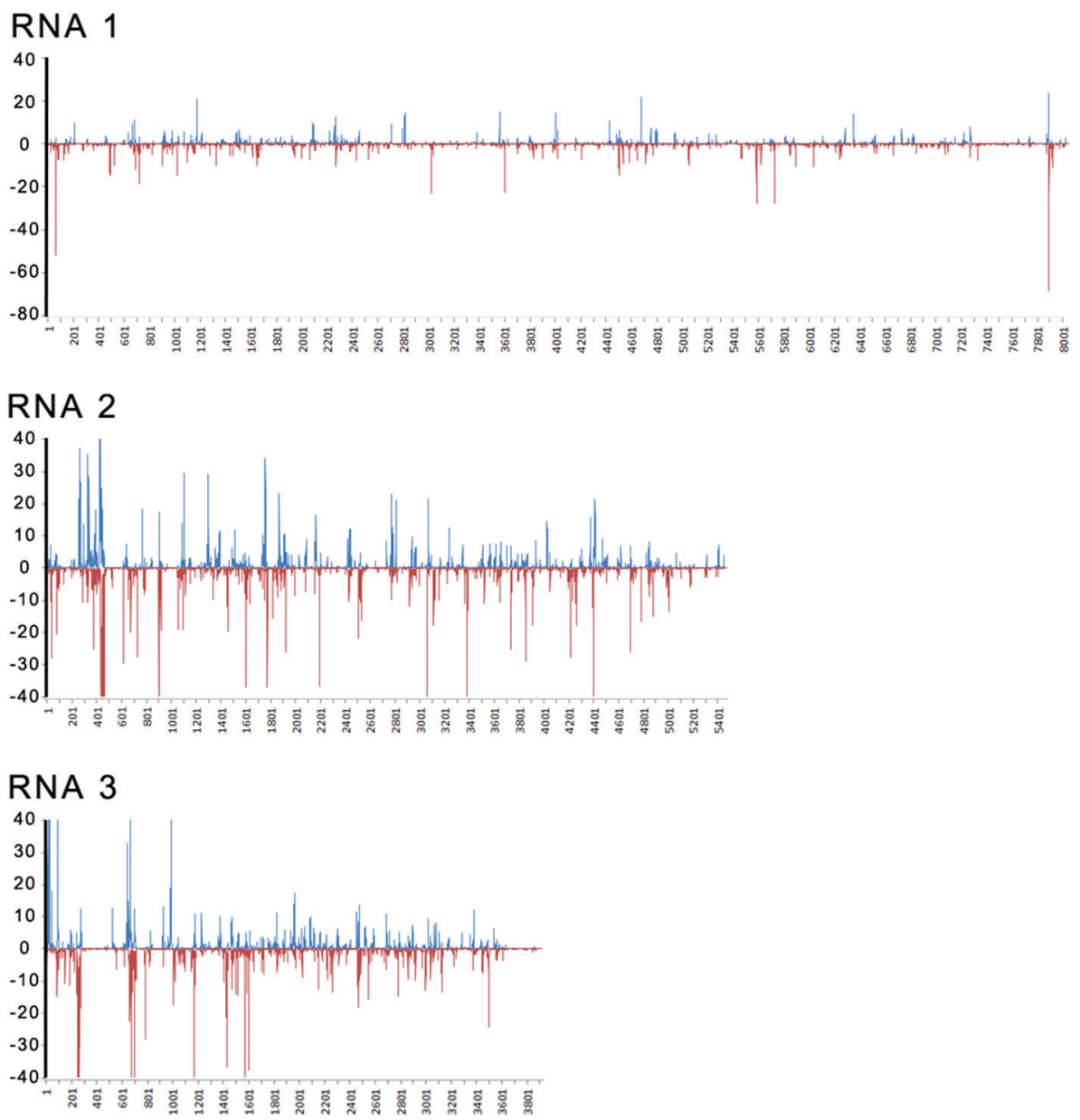

Fig. 5. Distribution along the three genomic RNAs of the sRNA reads (18-50 nt) to the PYVV present in infected potato sample M2 (S. phureja). Positive-sense RNAs are represented at the top of the charts (blue), whereas negative-polarity RNAs are represented at the bottom (red) in RPM. Genomic RNAs 2 and 3 generated more reads per unit of length than genomic RNA 1. In addition, some regions in each of the genomic RNAs generated more sRNA reads (hotspots) than others.

the genomic RNAs of PYVV infecting samples M2, M9, M14 and M19: in the single PYVV infections (samples M2, M9 and M19) the nucleotides that remained unidentified in those RNAs were few in number $(\sim 0.3 \%$ of the total for RNA 1 , $0.6 \%$ for RNA 2 and $2.8 \%$ for RNA 3). However, in the case of the mixed infection with PVY in sample M14, those percentages were higher (3.4\% for RNA 1, 2.8\% for RNA 2 and $12.8 \%$ for RNA 3), probably because of the reduced number of reads to PYVV sRNAs that were obtained (Fig. 3). Interestingly, in the four infections the unidentified nucleotides often mapped to the same segments in those genomic RNAs (Fig. 3, compare between unassembled regions of genomic RNAs, indicated by their nucleotide flanking numbers). This suggests that the absence of overlapping continua of sRNA reads in those genomic regions that prevent the assembly of contigs is also a stable feature conserved among different infections.

Comparison of the reconstructed RNA genomes showed very high identities among them $(>98.4 \%)$. The sequences of the reconstructed genomes were compared with sequences of other PYVV isolates from the GenBank database. The RNA 1 sequences of samples M14 and M19 showed high identity $(99.7 \%)$ with a PYVV sequence from an isolate from S. phureja (KR998193). The RNA 2 sequences of samples M9 and M19 were very similar $(>98.7 \%)$ to sequences from Colombia and Peru (MN088361 and AJ557129, respectively) and the RNA 3 sequences of samples M2, M9, M14 and M19 presented similarities higher than $98,6 \%$ with Colombian isolates from S. phureja (KR998195), S. lycopersicum (KX573903) and S. tuberosum from Peru (NC006061 and AJ508757). The PVY isolate recovered from sample M14 produced a $9718 \mathrm{nt}$ sequence (MT522445) comprising the full-length sequence. This sequence had more than $99.8 \%$ similarity with other PVY isolates from Colombia such as MK563993.1, MF176827.1 and KT336552.1.

We also studied the distribution of sRNA reads along the genomic RNAs of the PYVV and PVY genomes (Figs 5 and 


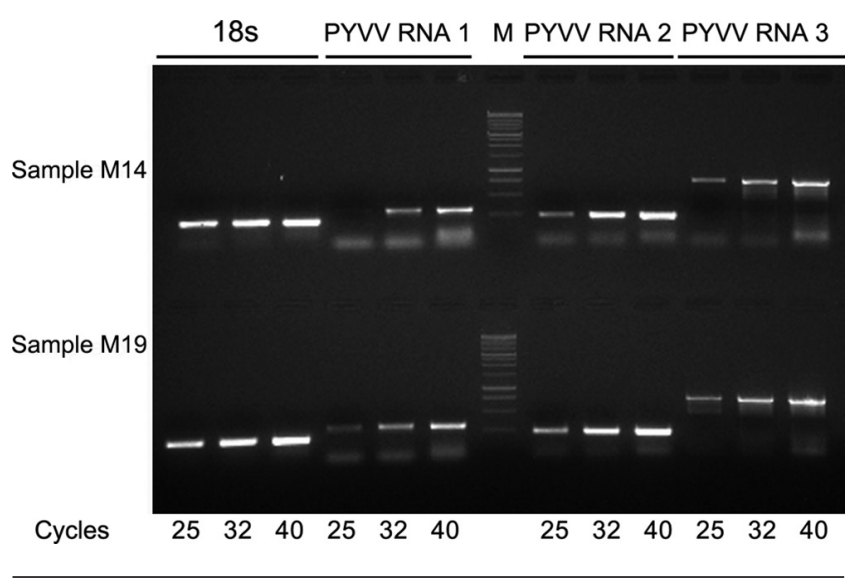

Fig. 6. Determination by semi-quantitative RT-PCR amplification of the relative levels of accumulation of PYVV in sample M14 (S. tuberosum) mixed infection with PVY potyvirus vs. sample M19 (single infection) (S. tuberosum). Fragments from each of the three PYVV genomic RNAs were amplified for 25, 32 and 40 cycles separately using appropriate oligonucleotides (shown in Table S1). A fragment of 18S rRNA was also amplified in the same way as the internal gene control. Amplification of the three genomic PYVV fragments, as well as of the $18 \mathrm{~S}$ fragment, was comparable among the two samples.

S2). The number of sRNA reads to each PYVV genomic RNA was not uniform: genomic RNAs 2 and 3 generated many more reads per unit of length than genomic RNA 1. Within each of the genomic RNAs, some regions generated more sRNA reads than others. These hotspots to sRNA formation were more evident in RNAs 2 and 3 (Fig. 5).

\section{Relative amounts of PYVV in a single vs. a mixed infection with the potyvirus PVY}

Assessment of the relative accumulation of each of the three genomic RNAs of PYVV using three sets of primers in a single infection vs. a mixed infection with PVY by semi-quantitative RT-PCR showed that the relative levels of crinivirus RNAs remained comparable in both cases and therefore a positive synergism effect of PVY on the titres of the crinivirus was not observed (Fig. 6, PYVV accumulation in samples M14 vs. M19).

\section{Characterization of infection symptoms in progenies raised under controlled greenhouse conditions from tubers of plants sampled in the fields}

Tubers from the 12 mother plants that had been sampled in the field were potted in the greenhouse, and symptoms in the progeny plants that were raised from them were monitored until those plants senesced. Tubers of $S$. phureja plants began to shoot $\sim 10$ days after being planted, while those of S. tuberosum plants began to produce leaves $\sim 4$ weeks after planting. In total, 22 tubers from plants with PYVD symptoms, atypical yellowing symptoms or non-symptomatic mother plants produced viable progeny plants. Symptoms were observed in nine of them at some point in their life cycle
(Table 2), while the rest did not show visually recognizable symptoms of viral infection. Some of the progeny plants that derived from the 12 mother plants that had shown typical PYVD symptoms or atypical yellowing symptoms in the field showed under our experimental conditions secondary and tertiary vein yellowing that never developed into the fully yellow leaves (Fig. S1) characteristic of PYVV-infected field plants (Table 2). Progeny plants were also tested for the presence of PYVV by RT-PCR (Table 2). In S. phureja the symptoms appeared during the third week post-emergence, but in one plant the symptoms were seen briefly for about 1 week and in another plant the symptoms disappeared in the seventh week. In the $S$. tuberosum plants, the symptoms were observed at about week 16 (Table 2) and remained present until senescence, 4 weeks later. In five of the daughter plants derived from infected plants, PYVV was not detected, indicating that the virus had not transmitted through those tubers, and suggesting a non-uniform distribution of virus in the plant tubers. Additionally, in four non-symptomatic cases, RT-PCR-positive daughter plants originated from symptomatic infected mother plants (Table 2).

\section{DISCUSSION}

Crinivirus infections often induce mild symptoms that in the presence of co-infecting viruses become more severe than those that are induced by either of those viruses separately $[11,20]$. This is the case for sweet potato plants infected with Sweet potato chlorotic stunt virus (SPCSV, genus Crinivirus) that show either absence of symptoms or mild symptoms, but when co-infected with members of the genera Potyvirus, Ipomovirus, Cucumovirus and Carlavirus develop severe diseases [21, 22]. Infections of cucumber by Cucurbit chlorotic yellows virus (CCYV) and Cucurbit yellow stunting disorder virus (CYSDV) criniviruses constitute another example: they cause symptoms in single infections that in co-infections with a Begomovirus become more severe, with crop yield reductions [23].

PYVD causes important losses to potato crops in the Andean region, and is associated ith PYVV. In this pathosystem, in addition to fully symptomatic plants, the existence of infected, asymptomatic field plants whose tuber production is not affected has previously been reported $[1,3,7]$. In addition, mixed viral infections involving PYVV appear to be very abundant in $S$. tuberosum fields in the Antioquia department of Colombia [24]. Whether observed differences in symptoms in infections by PYVV are caused by environmental factors, by the presence of other co-infecting viruses or by both is under investigation. To gain further insights into the nature and variations of PYVD and on the molecular properties of PYVV infections, we sampled leaves and tubers from potato fields at three locations in the Colombian Highlands and determined by HTS the sRNA populations present in several of those plants. This technique allows the identification and assembly of viromes infecting several plant species, in many cases leading to discoveries of undetected latent viruses and multiple infections [16, 24-27]. 


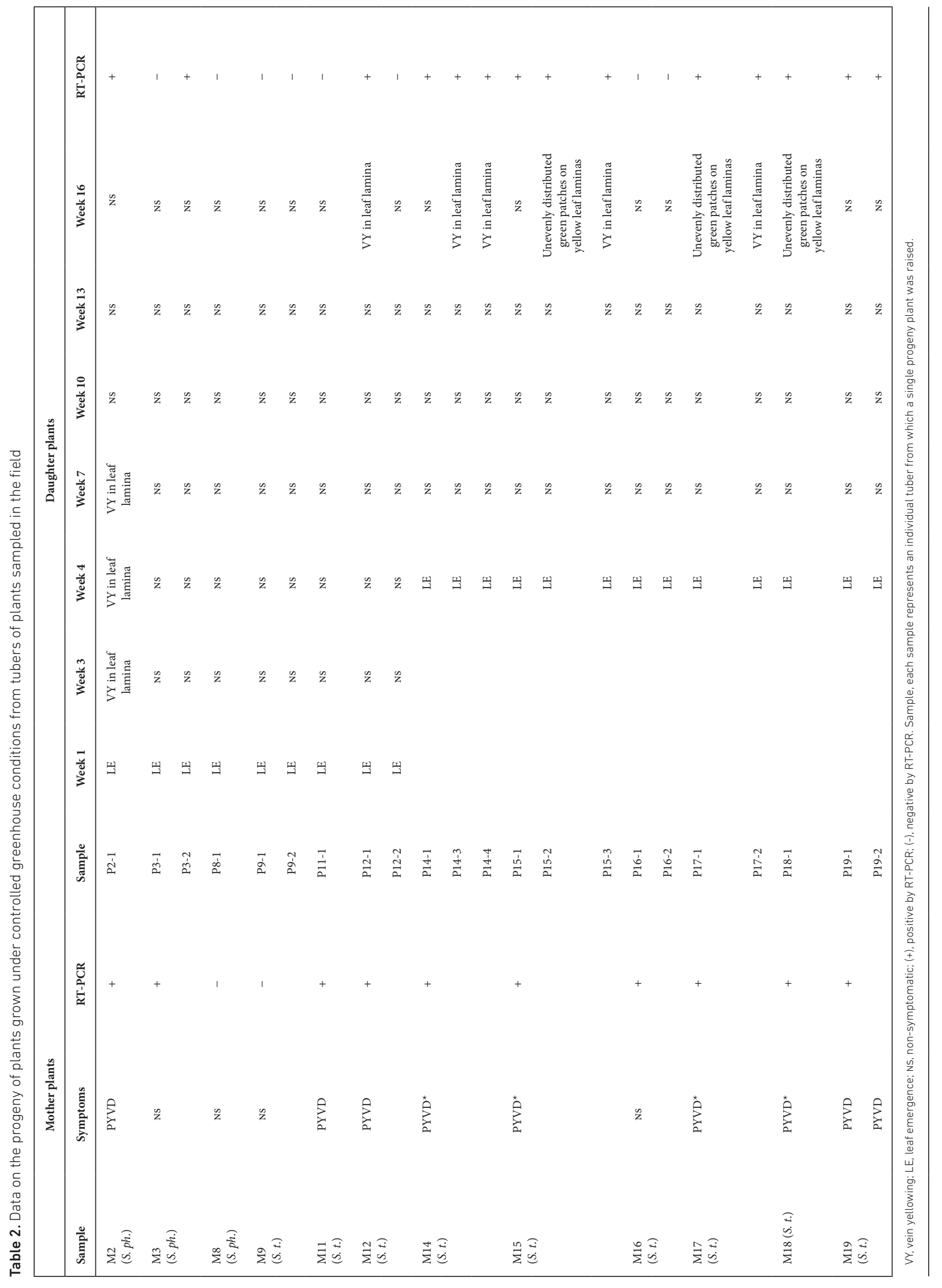


We sampled plants that displayed two types of yellowing symptoms, and also plants without symptoms (Table 1 and Fig. 1). We tested them for PYVV presence by RT-PCR (Table 1, Fig. 1), and the sRNA populations of five of them were determined by HTS. According to RT-PCR tests, four of those plants were infected with PYVV: three of the plants presented typical PYVD yellowing symptoms (samples M2, M9, M19), one showed atypical yellowing (M14), and one (M8) had no symptoms and was uninfected (Table 1). For each of the five samples, between 25 and 36 million total sRNA reads were obtained. In all cases the most abundant sRNAs were those of 21, 22 and $24 \mathrm{nt}$ in length. Reads were classified as of plant or non-plant origin (Fig. 2, pie charts of RPM). The vast majority of the non-plant reads matched viral sequences. They matched specifically to those of the three PYVV genomic RNAs [9] in plants displaying typical PYVD symptoms (samples M2, M9 and M19), and of PYVV and of the potyvirus PVY in the case of the sample that showed atypical yellowing symptoms (M14). Unlike other cases in which the presence of other viruses in addition to PYVV had been detected, in plants such as lulo (S. quitoense) [15] or potato (S. phureja) [27], in our case PYVV was the only RNA virus present in the three field potato plants displaying PYVD symptoms. Therefore, co-infecting viruses were not associated with PYVD symptoms in our sampled plants.

In sample M14 the relative proportion of the three PYVV genomic RNAs was only $1 \%$ of the total viral sRNA reads, whereas PVY sRNAs accounted for the remaining 99\% (Fig. 2, fourth pie chart from the left). This is more disproportionate than was previously reported ( $8 \%$ for PYVV and $92 \%$ for PVY in mixed infections in S. phureja) [16], although numbers of total sRNA reads per plant sample (0.2-1.2 million) in that study were at least 20 times lower than in this study. Our data support that PVY, a systemic virus not limited to the vasculature, competes strongly with the phloem-limited PYVV for the antiviral response of the plant RNA silencing machinery.

When we related the ratios of sRNA reads obtained to the expected lengths of the viral or plant template sequences that triggered them (RPKM) we found that PYVV genomic RNAs were highly immunogenic, indicating that they generated very large amounts of reads, relative to those generated by plant sequences, despite the former being confined to the phloem (Fig. 4, corresponding pie charts). Ratios of RPMK of the PYVV RNA 1 sequence were roughly one third of those of PYVV RNAs 2 or 3 sequences in the three plants that were singly infected with PYVV (M2, M9, M19), as well as in plant M14 with a PYVV and PVY co-infection (Fig. 4). These ratios appeared more similar among samples M14 and M19 (Fig. 4, fourth vs. fifth pie charts from the left), and among samples M2 and M9 (Fig. 4, first vs. third pie charts). Interestingly, sample pairs M2-M9, and M14-M19 shared geographical vicinity (Table 1, Fig. 1). These RPMK ratios may reflect the actual relative abundance of each of the PYVV viral genomic RNAs in the infected tissues sampled. However, this would only hold true if the three viral genomic RNAs had similar immunogenicity, and abilities to trigger sRNA synthesis by the plant silencing machinery. This is uncertain. Regardless, relative ratios of sRNA RPMK for each of the three genomic PYVV RNAs remained broadly constant among the four infections, even in the case of the sample co-infected with PVY, suggesting that the sRNA response to each viral RNA may be a conserved property of PYVV infections in potato.

Assemblies of the sRNA reads allowed the reconstruction of the sequences of the complete or almost complete genomic PYVV RNAs in the four infected samples (M2, M9, M14, M19; Fig. 3), and of the co-infecting PVY isolate in sample M14. In the case of single PYVV infections (M2, M9 and M19) some nucleotide segments were left unassembled, but they were few in number (constituting $~ 0.3 \%$ of the total for RNA $1,0.6 \%$ for RNA 2 and $2.8 \%$ for RNA 3 ). In the case of the mixed infection with the potyvirus PVY in sample M14, those percentages were higher (3.4\% for RNA 1, 2.8\% for RNA 2 and $12.8 \%$ for RNA 3), probably because of the 10 -fold reduced number of reads to PYVV sRNAs that was obtained from that sample (Fig. 3). As expected, the assembled PYVV sequences obtained showed high levels of identity (>98.4\%) with previously reported PYVV sequences from Colombia and Perú $[9,13-15,25,27]$. Interestingly, in the reconstructed contigs, unidentified nucleotide segments often mapped to the same regions of the genomic RNAs, which suggests that the absence of overlapping sRNA reads preventing assembly of contigs in those segments is a stable trait that is conserved between different infections (Fig. 3). We also mapped the distribution of positive or negative sRNA reads along the three PYVV genomic sequences (Fig. 5) and found that they were not uniformly distributed throughout the whole sequence: some regions were hotspots for sRNA formation. Therefore, we identified viral genomic regions that were hotspots for sRNA formation and others that were poor inducers of overlapping sRNAs by the plant silencing machinery (Figs. 3 and 5 ), possibly because of their differential conformational or structural accessibilities to the latter.

Synergistic effects on viral titres of co-infections of criniviruses with viruses of other genera have been reported in several pathosystems: for example, when Lettuce infectious yellows virus (LIYV, genus Crinivirus) co-infects Nicotiana benthamiana plants with Turnip mosaic virus (TuMV, genus Potyvirus), the result is a synergistic interaction in which the accumulation levels of LIYV is enhanced compared with titres of plants infected only with this virus. In this case, the expression of the silencing suppressor of TuMV HC-Pro protein seems to explain this observation [28]. In another well-studied case, mixed infections of the Crinivirus SPCSV and Sweet potato feathery mottle virus (SPFMV, genus Potyvirus) in sweet potato plants produce a severe disease called sweet potato virus disease (SPVD), whose symptoms are not produced when either of the viruses is present in single infections. In this case, however, the synergistic interaction favours the potyvirus increasing the coat protein titre of SPFMV up to 600 -fold, while the amount of SPCSV remains about the same or decreases slightly [21]. Co-infections of melon plants (cv. Piel de sapo) by the crinivirus CYSDV and the potyvirus Watermelon mosaic virus (WMV) led to marked increases 
in the titres of the crinivirus whereas those of the potyvirus decreased, when compared to single infections of melon plants by those viruses [29].

In our case, co-infection of phloem-limited PYVV and systemically infecting PVY (sample M14) produced a green and intense bright yellowing mosaic in sink areas of potato leaves (such as shown in Fig. 1b, lower panel to the right) that were different from the PYVD symptoms we found in plants infected with PYVV alone (samples M2, M9, M19). PVY on its own is a widespread virus in Colombian potato crops that usually produces mild mosaic symptoms; isolates belonging to the PYY ${ }^{\mathrm{NTN}}, \mathrm{PVYN}^{\mathrm{NTN}}$ and $\mathrm{PVY}^{\mathrm{O}}$ groups have been detected in Colombia [30] although we do not know which one is our particular isolate. In the interaction between PYVV and PVY in this host the effect of the mixed infections on PVY accumulation was not measured, but we found that the titre of the PYVV genomic RNAs was not altered in the mixed infection relative to the single infections (Fig. 6). This suggests that potential synergism effects on viral titres in co-infections of PYVV with potyviruses may be specific to the interacting viral pair, and also to the specific host species in which this co-infection is taking place.

Finally, studies were made on the tuber transmission of PYVV under semi-controlled glasshouse conditions. One interesting observation was that in our experimental conditions the characteristic symptoms of PYVV field-infected plants, such as intense yellow leaf laminas, were not observed. Instead, the most relevant symptom in the infected plants was secondary and tertiary vein yellowing that never developed into fully yellow leaves (Fig. S1). It is possible that milder or absent symptoms may relate to glasshouse conditions in which daughter plants were grown from field-collected tubers. It is noteworthy that conditions such as average temperature, relative humidity and exposure to solar radiation were considerably different between the glasshouse and the field in which mother plants developed. Differential symptoms could be attributed to the different geographical origins of the samples, but this is difficult to demonstrate because of the small number of samples analysed. Additionally, a common practice among the Colombian growers is sowing of seed-tubers of unknown origin bought in the market, instead of using the seed-tubers produced in their own fields. A previous work in which the variability of the PYVV CP gene was studied in five distant departments of Colombia showed that PYVV variants of one department could be found at a low frequencies in fields located in distant departments, probably due the extended traffic of seed-tubers between remote locations [31]. In two S. phureja plants, symptom remission was observed (Table 2), demonstrating that the occurrence of recovery phenotypes and asymptomatic infections in this species is possible. As had been shown previously, plants showing PYVV were able to transmit PYVV to daughter plants by vertical transmission through tubers [7], but in some cases infected mother plants gave rise to non-syntomatic progeny plants. In studies performed on Potato virus X (PVX, genus Potexvirus), Potato andean mottle virus (APMoV, Comovirus), PVY (genus Potyvirus) jointly with PVX and PLRV Potato leafroll virus (PLRV, genus Polerovirus), incomplete autoinfection was observed in all cases. It has been hypothesized that temperature is a major factor in affecting viral replication and translocation, and could account for these results [32]. It should also be taken into account that when we collected the tubers from the field, some mother plants were not completely mature and the tubers were in the filling process. It is possible that the virus had not yet been translocated and therefore not passed to daughter plants.

To date, the molecular determinants that might explain the presence or absence of PYVD symptoms in PYVV-infected plants are unknown, but our results show that no co-infecting viruses were involved in the symptom repression observed in non-symptomatic, PYVV-infected progeny plants, and suggest that the impact of environmental conditions could be relevant.

\section{Funding information}

This work was funded by Grants PID2019-109304RB-100 and COOPB20310 from the 2019 and 2017 calls by the Ministry of Science and Innovation of the Government of Spain, and the Spanish Council for Scientific Research; and by Grant CIAS 2742 from the Universidad Militar Nueva Granada, Colombia.

\section{Author contributions}

A.N. partcipated in the geographical survey of plants, virus detection, and analysis of HTS data and analysis of vertical transmission in progenies from surveyed plants. F.d.T. participated in the analysis of HTS data and in relative virus quantification among samples. F.T. particated in the editing and discussion of results for preparation of the manuscript. L.F. and T.C. participated in the highland survey of potato samples, laboratory extraction and virus detection and quantification, analysis of HTS data, and writing of the manuscript.

\section{Conflicts of interest}

The authors declare that there are no conflicts of interest

References

1. Salazar LF, Muller G, Querci M, Zapata JL, Owens RA. Potato yellow vein virus: its host range, distribution in South America and identification as a crinivirus transmitted by Trialeurodes vaporariorum. Ann Appl Biol 2000;137:7-19.

2. Salazar LF. Emerging and re-emerging potato diseases in the Andes. Potato Res 2006;49:43-47.

3. Guzmán-Barney M, Franco-Lara L, Rodríguez D, Vargas L, Fierro JE. Yield losses in Solanum tuberosum Group Phureja cultivar Criolla Colombia in plants with symptoms of PYVV in field trials. Am J Potato Res 2012;89:438-447.

4. Rincón DF, Vásquez DF, Rivera-Trujillo HF, Beltrán C, Borrero-Echeverry F. Economic injury levels for the potato yellow vein disease and its vector, Trialeurodes vaporariorum (Hemiptera: Aleyrodidae), affecting potato crops in the Andes. Crop Prot 2019:119:52-58.

5. Machida-Hirano R. Diversity of potato genetic resources. Breed Sci 2015;65:26-40.

6. Spooner DM, Ghislain M, Simon R, Jansky SH, Gavrilenko T Systematics, diversity, genetics, and evolution of wild and cultivated potatoes. Bot Rev 2014;80:283-383

7. Guzmán-Barney M, Hernández AK, Franco-Lara L. Tracking foliar symptoms caused by tuber-borne Potato Yellow Vein Virus (PYVV) in Solanum Phureja (Juz et Buk) Cultivar "Criolla Colombia. Am J Potato Res 2013;90:284-293.

8. Franco-Lara L, Rodríguez D, Guzmán-Barney M. Prevalence of Potato yellow vein virus (PYVV) in Solanum tuberosum Group Phureja Fields in Three States of Colombia. Am J Potato Res 2013:90:324-330. 
9. Livieratos IC, Eliasco E, Müller G, Olsthoorn RCL, Salazar LF. Analysis of the RNA of Potato yellow vein virus: Evidence for a tripartite genome and conserved 3 '-terminal structures among members of the genus Crinivirus. J Gen Virol 2004;85:2065-2075.

10. Martelli GP, Agranovsky AA, Bar-Joseph M, Boscia D, Candresse T. Family Closteroviridae. King A, Adams M, Carstens E and Lefkowitz E (eds). In: Virus taxonomy. Ninth report of the International Committee on Taxonomy of viruses. Amster- dam: Elsevier-Academic Press; 2011. pp. 987-1001.

11. Tzanetakis IE, Martin RR, Wintermantel WM. Epidemiology of criniviruses: An emerging problem in world agriculture. Front Microbiol 2013:4:1-15.

12. Karasev AV. Genetic diversity and evolution of closteroviruses. Annu Rev Phytopathol 2000;38:293-324.

13. Álvarez D, Gutiérrez $P$, Marín M. Secuenciación del genoma del Potato yellow vein virus (PYVV) y desarrollo de una prueba molecular para su detección. Bioagro 2017;29:3-14.

14. Muñoz Baena L, Gutiérrez Sánchez PA, Marín Montoya M. Secuenciación del genoma completo del Potato yellow vein virus (PYVV) en tomate (Solanum lycopersicum) en Colombia. Acta biol Colomb 2017;22:5.

15. Gallo Y, Toro LF, Jaramillo H, Gutiérrez PA, Marín M. Identificación y caracterización molecular del genoma completo de tres virus en cultivos de lulo (Solanum quitoense) de Antioquia (Colombia). Rev Colomb Cienc Hortíc 2018;12:281-292.

16. Villamil-Garzón A, Cuellar WJ, Guzmán-Barney M. Natural co-infection of Solanum tuberosum crops by the Potato yellow vein virus and potyvirus in Colombia. Agron colomb 2014:32:213-223.

17. Langmead B, Trapnell C, Pop M, Salzberg SL. Ultrafast and memory-efficient alignment of short DNA sequences to the human genome. Genome Biol 2009;10:R25

18. Potato Genome Sequencing Consortium, Xu X, Pan S, Cheng S, Zhang B, et al. Potato Genome Sequencing Consortium. Genome sequence and analysis of the tuber crop potato. Nature 2011:475:189-195.

19. Zheng Y, Gao S, Padmanabhan C, Li R, Galvez M. VirusDetect: an automated pipeline for efficient virus discovery using deep sequencing of small RNAs. Virology 2017;500:130-138.

20. Varvara I, Maliogka VI, Wintermantel WM, Orfanidou CG, Katis NI. Criniviruses infecting vegetable crops. In applied plant biotechnology for improving resistance to biotic stress. Academic Press, 2020, pp. 225-250.
21. Karyeija RF, Kreuze JF, Gibson RW, Valkonen JPT. Synergistic interactions of a potyvirus and a phloem-limited crinivirus in sweet potato plants. Virology 2000;269:26-36.

22. Untiveros M, Fuentes S, Salazar L. Synergistic interaction of Sweet potato chlorotic stunt virus (Crinivirus) with Carla-, Cucumo-, Ipomo-, and Potyviruses Infecting Sweet Potato. Plant Dis 2007:91:669-676.

23. Abrahamian P, Sobh H, Seblani R, Abou-Jawdah Y. Co-infection of two Criniviruses and a Begomovirus enhances the disease severity in cucumber. Eur J Plant Pathol 2015;142:521-530.

24. Golyaev V, Candresse T, Rabenstein F, Pooggin MM. Plant virome reconstruction and antiviral RNAi characterization by deep sequencing of small RNAs from dried leaves. Sci Rep 2019:9:1-10.

25. Gallo García Y, Sierra Mejía A, Donaire Segarra L, Aranda MA, Gutiérrez PA. Natural coinfection of RNA viruses in potato (Solanum tuberosum subsp. andigena) crops in Antioquia (Colombia. Acta Biol Colomb 2019:24:546-560.

26. Roossinck MJ, Martin DP, Roumagnac P. Plant virus metagenomics: Advances in virus discovery. Phytopathology 2015;105:716-727.

27. Sierra Mejía A, Gallo García Y, Estrada Arteaga M, Gutiérrez AP, Marín Montoya M. Molecular Detection of Six RNA Viruses in Tuber Sprouts of Potato. (Solanum phureja) in Antioquia, Colombia. Bioagro, 2020, pp. 3-14.

28. Wang J, Turina M, Medina V, Falk BW. Synergistic interaction between the Potyvirus, Turnip mosaic virus and the Crinivirus, Lettuce infectious yellows virus in plants and protoplasts. Virus Res 2009:144:163-170.

29. Domingo-Calap ML, Moreno AB, Díaz Pendón JA, Moreno A Fereres $A$. Assessing the impact on virus transmission and insect vector behavior of a viral mixed infection in melon. Phytopathology 2019:110:174-186.

30. Muñoz ED, Gutiérrez SP, Marín MM. Detection and genome characterization of Potato virus $Y$ isolates infecting potato (Solanum tuberosum L.) in La Union (Antioquia, Colombia). Agron Colomb 2016:34:317-328.

31. Rodríguez PA, Franco-Lara L, Guzmán Barney M. Inter and intra variation of Potato yellow vein virus in Three Potato Species from Colombia. Rev Fac Nal Agr Medellín 2015;68:7387-7398.

32. Bertschinger L, Bühler L, Dupuis B, Duffy B, Gessler C. Incomplete infection of secondarily infected potato plants - an environment dependent underestimated mechanism in plant virology. Front Plant Sci 2017:8:1-13.

\section{Five reasons to publish your next article with a Microbiology Society journal}

1. The Microbiology Society is a not-for-profit organization.

2. We offer fast and rigorous peer review - average time to first decision is 4-6 weeks.

3. Our journals have a global readership with subscriptions held in research institutions around the world.

4. $80 \%$ of our authors rate our submission process as 'excellent' or 'very good'.

5. Your article will be published on an interactive journal platform with advanced metrics.

Find out more and submit your article at microbiologyresearch.org. 